\title{
Searching for Gravitational Waves from the Inspiral of Precessing Binary Systems: Problems with Current Waveforms.
}

\author{
Philippe Grandclément* and Vassiliki Kalogera ${ }^{\dagger}$ \\ Northwestern University, Dept. of Physics 83 Astronomy, 2145 Sheridan Road, Evanston 60208, USA \\ Alberto Vecchio $\ddagger$ \\ School of Physics and Astronomy, The University of Birmingham, Edgbaston, Birmingham B15 2TT, UK
}

(Dated: November 22, 2002)

\begin{abstract}
We consider the problem of searching for gravitational waves emitted during the inspiral phase of binary systems when the orbital plane precesses due to relativistic spin-orbit coupling. Such effect takes place when the spins of the binary members are misaligned with respect to the orbital angular momentum. As a first step we assess the importance of precession specifically for the firstgeneration of LIGO detectors. We investigate the extent of the signal-to-noise ratio reduction and, hence, detection rate that occurs when precession effects are not accounted for in the template waveforms. We restrict our analysis to binary systems that undergo the so-called simple precession and have a total mass $\lesssim 10 M_{\odot}$. We find that for binary systems with rather high mass ratios (e.g., a $1.4 \mathrm{M}_{\odot}$ neutron star and a $10 \mathrm{M}_{\odot}$ black hole) the detection rate can decrease by almost an order of magnitude. Current astrophysical estimates of the rate of binary inspiral events suggest that LIGO could detect at most a few events per year, and therefore the reduction of the detection rate even by a factor of a few is critical. In the second part of our analysis, we examine whether the effect of precession could be included in the templates by capturing the main features of the phase modulation through a small number of extra parameters. Specifically we examine and tested for the first time the 3-parameter family suggested in [1]. We find that, even though these "mimic" templates improve the detection rate, they are still inadequate in recovering the signal-to-noise ratio at the desired level. We conclude that a more complex template family is needed in the near future, still maintaining the number of additional parameters as small as possible in order to reduce the computational costs.
\end{abstract}

\section{INTRODUCTION}

With the international network of ground-based gravitational wave (GW) detectors LIGO [2], VIRGO [3], GEO600 [4], TAMA [5] coming online, the need for accurate source modeling, which can guide the construction of optimal data analysis strategies and therefore maximizes the detection efficiency is becoming increasingly pressing. The expected signals will be so weak compared to the intrinsic noise of the detectors, that one relies on a number of different data-processing techniques for signal extraction and detection, followed by search for coincidences in two or more instruments. Matched-filtering provides the optimal linear search technique [6,7], particularly suitable to search for signals that are characterized by a large number of wave cycles within the interferometer observational window $(\simeq 40 \mathrm{~Hz}-2 \mathrm{kHz})$, and whose waveforms can be accurately modeled from a theoretical point of view. A well-known example of such a signal is the binary inspiral of compact objects, either neutron stars (NS) or stellar-mass black holes (BH). Such inspiral events are the most promising candidates for ground-based interferometers, and, for the instruments of first generation, especially those with high total mass $(\mathrm{BH} / \mathrm{NS}$ and/or $\mathrm{BH} / \mathrm{BH}$ binary systems), see $[8]$ and references therein.

A crucial component in the successful implementation of matched-filtering is the construction of a reliable family of gravitational waveforms to be used as "templates". As one might expect intuitively, the closer the templates are to the "true" signal, the higher the signal-to-noise ratio (SNR) at which a detection can be achieved. For this reason in recent years a tremendous effort has been devoted to the computation of the waveforms that characterize the inspiral phase, using different approaches, such as post-Newtonian expansions (see [9] and references therein for an extensive review) and Padé approximants [10, 11].

The simplest family of templates corresponds to GW signals from two point-masses orbiting each other. However, it is possible that the true inspiral is characterized by other more complicated effects, such as relativistic spin-orbit

\footnotetext{
*PGrandclement@northwestern.edu

†vicky@northwestern.edu

$\ddagger$ av@star.sr.bham.ac.uk
} 
and spin-spin couplings due to the presence of high spins misaligned one with respect to the other and to the orbital angular momentum. One of the most dramatic effects induced by such coupling is the precession of the binary orbital plane around a fixed direction, with a significant number of precession cycles within the GW frequency band of interest. As a result of this precession the polarization of the waves impinging on the detector changes during the observation, which in turn produces an amplitude and phase modulation of the signal at the detector output [12]. It is well known that if such modulation is not accounted for in the search templates the detection efficiency could decrease significantly, especially for binaries containing BHs and/or characterized by large mass ratios [1, 13]. This would lead to a reduction of the volume of the Universe accessible by any given search and hence of the rate of detection. Current predictions of the detection rates for binary inspiral with the first-generation of laser interferometers are relatively low, in the most optimistic cases up to just a few events per year even for massive binaries with two black holes [14, 15]. Such estimates are obtained by assuming perfect match between the signal and, at least, one of the templates of the filter bank. If modulations induced by spin-orbit precession are not included into the family of templates, the actual signal-to-noise ratio that can be achieved in any given search is lower. If such a loss of SNR occurs for a significant portion of the parameter space that characterize binary systems, the chance of detections by LIGO and other ground-based interferometers would be severely compromised. This would in turn require to construct a new family of template that takes precession effects into account. In the case of binaries with two neutron stars, both observations of pulsar spin periods $[16,17]$ and our current theoretical understanding of neutron star tidal evolution $[18,19]$ suggest that the spins are essentially negligible in neutron star binary systems. Therefore, we concentrate on binaries with either a black hole and a neutron star or two low-mass black holes.

The PN equations of motion, up to second post-Newtonian order, including spin-orbit and spin-spin corrections, have been derived in [20]. However, in this paper, instead of integrating the complete set of equations, we use an analytical approximation of the 1.5PN order equations derived by Apostolatos et al. [12, 13]. This approximation is valid in the regime of simple precession, which is relevant in the following two cases : (i) two spinning objects of equal mass $\left(m_{1}=m_{2}\right)$ or (ii) unequal-mass objects but with only the most massive one spinning $\left(S_{2}=0\right)$. Here we restrict our computations to systems for which only the most massive object is spinning. In the case of binaries with a black hole and a neutron star, such an assumption is physically well justified based on the same arguments relevant for binary neutron stars. In the case of binary black holes, the most massive of the two is also expected to be spinning more rapidly, since it is the one that in most cases is formed first, and therefore may have been spun up through accretion from its non-degenerate companion, the progenitor of the second black hole [15]. We restrict ourselves to systems with relatively moderate total mass (i.e. $m_{1}+m_{2} \lesssim 10 M_{\odot}$ ) so that one expects the PN-expansions to be fairly accurate [21]. The detectability of two, high mass, non-spinning black holes, for which the PN-expansions break down, has been recently assessed in [22]. Then, we consider whether the inspiral detection efficiency could be improved by using a new family of "mimic" templates, initially suggested by Apostolatos in [1] as a possible solution for the poor fitting factor achieved by non precessing templates. This new class of templates depends only on a small number of additional parameters - three in this case - but attempt to "mimic" the effect of precession. Apostolatos presented heuristic arguments supporting the choice of waveform: here we quantitatively explore for the first time whether they indeed recover most of the signal to noise ratio which is lost due the modulation effects. The result of this investigation is actually that this new family of templates is still inadequate to search for precessing binaries over essentially the entire parameter space.

This paper is organized as follows: Sec. II presents briefly the simple precession regime and reviews the various types of templates used throughout this paper, (post)-Newtonian and the "mimic" templates which include a correction term to the phase. The concept of the fitting factor is also introduced. Results are shown in Sec. III. We draw conclusions and discuss lines for future work in Sec. IV.

\section{ADOPTED SIGNALS AND TEMPLATES}

\section{A. Simple precession formalism}

Apostolatos et al. [12] have obtained a semi-analytical approximation of the waveforms to 1.5PN order, called simple precession. This approximation applies to a wide range of astrophysically relevant scenarios and is adopted here. The key effect of precession is to change the orientation of the orbital plane during the observation, and therefore introduce an amplitude and phase modulation in the signal recorded at the detector output due to the time-varying response of the detector to the two different polarizations of the GW. The effects of precession give rise to the following waveform :

$$
\tilde{h}_{\text {prec }}(f)=\Lambda(f) \tilde{h}(f)=\operatorname{AM}(f) \operatorname{PM}(f) \tilde{h}(f) .
$$

$\tilde{h}(f)$ is the waveform without precession, $\Lambda(f)$ represents the modulation factor of the waveform due to the change of relative orientation of the detector with respect to the binary orbital plane. $\Lambda$ can be decomposed as an amplitude 
modulation AM and a phase modulation PM. The simplicity of this regime is related to the fact that there is an analytical solution for both AM (Eq. (11) of [13]) and PM (Eq. (14) of [13]). More details can be found in [12, 13], but here we only outline the key features of the simple precession regime. We focus on binaries where only the most massive object $\left(m_{1}\right)$ carries a spin $\left(S=S_{1}\right)$. As explained in the introduction this case is physically the most relevant based on our astrophysical understanding of the formation of binary compact objects [15].

The key feature of simple precession is that the direction of the total angular momentum $\mathbf{J}$ remains essentially constant throughout the whole inspiral; the orbital momentum $\mathbf{L}$ and the spin $\mathbf{S}$ are locked together and precess at the same rate around $\mathbf{J}$ with constant tilt angle. We also note that throughout our calculations, as a first step, we do not include the Thomas precession phase (Eq. (14) in [13]).

Even though the expressions of the modulations AM and PM are not given in this paper, it is necessary to review the parameters on which they depend. The position and orientation of the binary with respect to the detector depends on four angles. The position of $\mathbf{L}$ on its precession cone involves another angle (see Fig. (4) of [12] for example). Notice that those five angles are randomly distributed. One also needs to give the constant magnitude of the spin $S$ and the cosine of the misalignment angle $\kappa$. The last two parameters on which the modulation $\Lambda$ depends are the two masses $m_{1}$ and $m_{2}$. To summarize, the modulation function depends on nine parameters, five of them being random angles. This large number of parameters needed to model the precession modulation makes it very unlikely, in the foreseeable future, to use the full precessing waveforms as templates for matched filtering, due to the enormous computational burden involved in such a search.

\section{B. Non-precessing waveforms}

The simplest way of describing the binary inspiral without precession, is to treat the objects as point masses in Newtonian gravity, and to determine the radiation by the quadrupole formula. By doing so, and applying the stationary phase approximation to transform the signal from the time to the frequency domain, one obtains the well known chirp signal [23-25] :

$$
\tilde{h}_{0}(f)=\mathcal{A} f^{-7 / 6} \exp \left(i \phi_{0}(f)\right) \quad ; \quad \phi_{0}(f)=2 \pi t_{c} f-\phi_{c}-\frac{\pi}{4}+\frac{3}{128}(\pi \mathcal{M})^{-5 / 3} f^{-5 / 3} .
$$

In Eq. (2), $\mathcal{M} \equiv\left(m_{1}^{3} m_{2}^{3} /\left(m_{1}+m_{2}\right)\right)^{1 / 5}$ is the chirp mass, $t_{c}$ is the coalescence time, $\phi_{c}$ the phase at coalescence and $\mathcal{A}$ a constant amplitude, which depends on the source distance, mass, location in the sky and orientation of the orbital plane. The Newtonian non-precessing waveforms constitute a 3 -parameter family $: t_{c}, \phi_{c}$ and $\mathcal{M}$. For the searches, however, it has be shown that the maximization over the parameter $\phi_{c}$ is analytical $[1,13]$ and a maximization over only two parameters is needed.

Post-Newtonian corrections to the phase of (2) can also be included. The exact expressions are given by Eqs. (3) of [1]. The PN corrections remove the degeneracy of the two masses into the single chirp mass parameter. The total number of parameters in this case is four: $t_{c}, \phi_{c}$ and the two masses $m_{1}$ and $m_{2}$.

The waveform (2) can either be used as non-precessing part of the signals ( $\tilde{h}$ in Eq. (1)) or as templates. In the latter case, when PN corrections are included, as the templates used for the real searches do not include spin effects, we set both the spin-orbit and spin-spin coupling terms to zero $(\beta=0$ and $\sigma=0$ in Eqs. (3) of [1]).

Let us mention that it is crucial to maintain the same PN-order for the non-precessing part of both the signal and the templates. Indeed, we want to ensure that whatever decrease in detection rate we calculate is related solely to precession effects and not from mismatch in the $\mathrm{PN}$-order of the non-precessing parts (this consistency requirement was not satisfied in [1]). In the course of our calculations we check that the inclusion of higher order corrections does not significantly modify the results obtained when considering non-precessing parts of Newtonian order only.

\section{C. "Mimic" templates}

To alleviate the problem of the large number of template parameters, Apostolatos [1] suggested the use of a new family of templates that still depends 3 extra parameters (beyond those associated with the non-precessing parts) and might be able to capture the main features of the precession modulation. He provided heuristic arguments that tends to suggest that this new class of templates could be effective in searching for precessing binaries, but never carried out a detailed quantitative analysis. The main effect of precession is to introduce an oscillatory modulation, for both the phase and the amplitude of the waveforms, which is completely different from the secular evolution of the GW phase. As the technique of matched-filtering rely on the template to stay in phase with signal over the frequency region where most of the SNR is accumulated, phase corrections are more crucial than amplitude corrections. As a 
first step in improving the filters, Apostolatos [1] suggested the inclusion of an oscillatory term to the phase $\phi$ of the templates:

$$
\tilde{h}_{\text {cor }}(f)=\mathcal{A} f^{-7 / 6} \exp \left(i \phi(f)+i \phi^{\text {cor }}(f)\right) \quad ; \quad \phi^{\text {cor }(-2 / 3)}=\mathcal{C} \cos \left(\mathcal{B} f^{-2 / 3}+\delta\right) .
$$

$\phi$ denotes the phase of the non-precessing waveform. If we consider only Newtonian order then $\phi$ is only $\phi_{0}$ of Eq. (2). We call the templates with such an additional term the mimic templates, because they represent an attempt to reproduce the main behavior of precession, and hopefully increase the detection rate. The mimic templates have three additional parameters $\mathcal{C}, \mathcal{B}$ and $\delta$, with the exponent of the dependence on the frequency being fixed. The choice of the exponent $-2 / 3$ comes from approximate formulae for the precession angle (cf. Eqs(29) of [13]). Indeed the value of the pulsation caused by precession relates closely to the number of precession cycles. Originally the choice of the exponent $-2 / 3$ was made by assuming that precession was only important for high values of $S$. However,we will see that, as stated in [1], the choice of the exponent is not crucial and one can, instead of $-2 / 3$, use the other approximate value -1 (cf. Eqs $(29)$ of [13]): $\phi^{\operatorname{cor}(-1)}=\mathcal{C} \cos \left(\mathcal{B} f^{-1}+\delta\right)$.

Considering only non-precessing parts of Newtonian order, we are left with five parameters : $\mathcal{M}, t_{c}$ and the three additional parameters $\mathcal{C}, \mathcal{B}$ and $\delta$. Performing the maximization in the full 5-parameter space is beyond what our computational resources allow. Fortunately, a simplification stems from the fact that the two chirp parameters $\mathcal{M}$ and $t_{c}$ are essentially uncorrelated with those of the mimic correction [1]. This implies that, instead of doing a maximization over 5 parameters, one can first maximize over the two parameters $\mathcal{M}$ and $t_{c}$ and then over the last three. This approach greatly reduces the computational time.

\section{The fitting factor}

Matched-filtering techniques are based on correlating the output of the detector with a family of pre-calculated templates. The highest possible SNR is then achieved when the signal $W$ coincides with one of the templates. In practice, the signal is unknown and one has to perform a search using a family of templates $T$ that depend on a given set of unknown parameters $\lambda_{1}, \lambda_{2}, \ldots$. Let us denote such a family with $T_{\vec{\lambda}}$. The fact that the exact signal is not included in the template family implies a loss in SNR

$$
\left(\frac{S}{N}\right)=\max _{\vec{\lambda}}\left[\frac{\left(W \mid T_{\vec{\lambda}}\right)}{\sqrt{\left(T_{\vec{\lambda}} \mid T_{\vec{\lambda}}\right)}}\right]=\mathrm{FF} \times\left(\frac{S}{N}\right)_{\max }=\mathrm{FF} \times(W \mid W)^{1 / 2},
$$

where $(\cdot \mid \cdot)$ is the scalar product of two waveforms, weighted by the noise of the detector (Eq. (2.3) of [26]; see also $[1,6,13,27]$ and references therein for more details). The maximum SNR is given by $(W \mid W)^{1 / 2}$. The fitting factor FF represents the loss of signal-to-noise ratio due to the fact that the signal and the templates do not exactly overlap (note that $0 \leq \mathrm{FF} \leq 1$ ). By definition, the fitting factor is expressed by :

$$
\mathrm{FF}=\max _{\vec{\lambda}}\left[\frac{\left(T_{\vec{\lambda}} \mid W\right)}{\sqrt{\left(T_{\vec{\lambda}} \mid T_{\vec{\lambda}}\right)(W \mid W)}}\right] .
$$

In all our work, contrary to $[1,13]$, we use to noise curve of the initial configuration of LIGO. The low-frequency cut-off $(40 \mathrm{~Hz})$ is at higher frequency that for advanced LIGO $(10 \mathrm{~Hz})$, and therefore the effects of precession may be reduced (fewer precession cycles in the band of the detector).

An important feature of the fitting factor is that it is independent of the absolute normalization of the signal, the templates and the noise. Should the templates, the signal or the noise be multiplied by any constant, the value of FF would not change. However such scalings change the value of $(S / N)_{\max }$ and therefore of $S / N$. It implies, for example, that the $\mathrm{FF}$ does not depend on the constant $\mathcal{A}$ of Eqs. (2).

Since we are interested in results that are independent of the specific values of the random angles, we decide to show the FF averaged over a given number of configurations of the random angles (chosen from a uniform distribution in solid angles). We note $S / N$ implies a reduction, by the same amount, of the maximum distance at which a source can be detected with a given signal-to-noise ratio. The total accessible volume therefore decreases by a factor of $<\mathrm{FF}>^{3}$. Assuming that sources are homogeneously distributed in space, then the detection rate is decreased by that same factor $<\mathrm{FF}>^{3}$. 


\section{RESULTS}

\section{A. Computational parameters}

The determination of the FF requires a maximization over the template parameters. Such maximization could be done by using sophisticated algorithms but it turns out that the dependency of FF on the parameters is rather complicated (see Fig. 2 of [13]). The presence of several local maxima could lead to convergence difficulties. In this paper we adopt a more straightforward way, which we have tested exhaustively. For each parameter of the templates, we determine a range of values needed for the search and populate such interval with discrete values of the parameters. Then, the FF is computed for all sets of parameters, allowing us to determine the maximum throughout the explored space. The smoothness of our curves is evidence in support of our assessment that we do find the global maxima.

For each parameter, the interval of search for the maximum is obtained by a trial search, with a very large interval, for a case of strong precession (cases of weaker precession require narrower ranges, but we still use the limits determined by the worst cases of strong precession). The number of templates used to populate this interval is calculated to ensure that the accuracy of $\mathrm{FF}$ is always below $10^{-2}$ (typically $30-100$ per parameter), based on convergence studies. Similarly we determine the appropriate number of collocation points $(1,000)$ for the computation of the cross-correlation integrals, and the number of sets of random angles $(2,000)$ used to perform the angle average. We note that the grids of the chirp mass and the total mass are logarithmic (because powers of the masses appear in the expressions for the templates).

\section{B. Importance of precession for detection rates}

We have calculated the angle-averaged fitting factor $<\mathrm{FF}>$ for various sets of masses $((3,3),(6,5),(7,3)$ and $(10,1.4)$ solar masses) and for the full range of spin magnitude (from 0.1 to 1 of the maximum value with steps of 0.1 ) and misalignment angle (from -1 to 1 for the cosine of the angle $\kappa$ with steps of 0.2 ). In favor of briefness, we present here the results for the cases of strongest precession : $m_{1}=10 M_{\odot}, m_{2}=1.4 M_{\odot}$ and for a subset of spin parameters. Indeed, as expected, the effects of precession are stronger for higher mass ratios [1].

The left panel of Figure 1 shows the reduction in detection rate (i.e. $<\mathrm{FF}\rangle^{3}$ ), as a function of $\kappa$, for various values of the spin magnitude of the most massive object in the binary. We have also chosen a couple of specific cases to examine our initial conjecture that PN effects would not change significantly the values of the $<\mathrm{FF}>$, as long as they are added consistently in the signal and the templates. The solid triangles show the rate reduction obtained when the $2 \mathrm{PN}$ correction to the phase of Eq. (2) is included, for $S=1$. In Figure 1 (left panel) we show the largest difference we have found, while for all other mass pairs PN and Newtonian points essentially overlap. Based on these small differences and our limited computational resources, we have mostly used the Newtonian signals and templates, for the non-precessing part of the waveforms. We note that for the other pairs of masses the maximum rate reduction lies in the range $0.3-0.6$.

Figure 1 shows clearly that the detection rate could be very significantly reduced, up to an order of magnitude. More precisely, the detection rate drops by more than a factor of two for almost all values of the spin magnitude, and virtually for all values of the misalignment angle (i.e. all values of $\kappa$ ), even for angles as small as for $\kappa=0.8$. Given the low expected detection rates for $\mathrm{BH}-\mathrm{NS}$ and $\mathrm{BH}-\mathrm{BH}$ binaries for the first-generation of ground-based detectors $[14,15]$, if spin-induced modulations effects are not included into the templates the chance of detection of BH-NS and BH-BH binary systems is likely to be drastically reduced. This justifies the quest for a new and more effective template family.

Our results can also be parameterized as a function of the mass of the binary system. The right panel of Fig. 1 shows the variation of $<\mathrm{FF}>^{3}$ with respect to $m_{1}$ (most massive, spinning object), maintaining the other mass fixed at $m_{2}=1.4 M_{\odot}$. Three different sets of spin properties are shown. Precession effects become more important as $m_{1}$ increases, and therefore the number of precession cycles within the relevant frequency band increases. As a result the detection rate drops by factors of 5 to 10 for high values of $m_{1}$. One can also see that the detection rate drops rather rapidly initially and then saturates. Assuming that detection is possible as long as $<\mathrm{FF}>^{3}$ is greater than a given threshold, say 0.5 , one can see that binaries with $m_{1}>4 M_{\odot}$ could remain undetected, showing once again the need for templates that account for modulation effects.

\section{Efficiency of the "mimic" templates}

Figure 2 shows the improvement in terms of $<\mathrm{FF}>$ (left panel) and $<\mathrm{FF}\rangle^{3}$ (right panel) achieved by including the "mimic" correction to the phase (Eq. (3)). The results are shown for two different choices for the exponent presented 

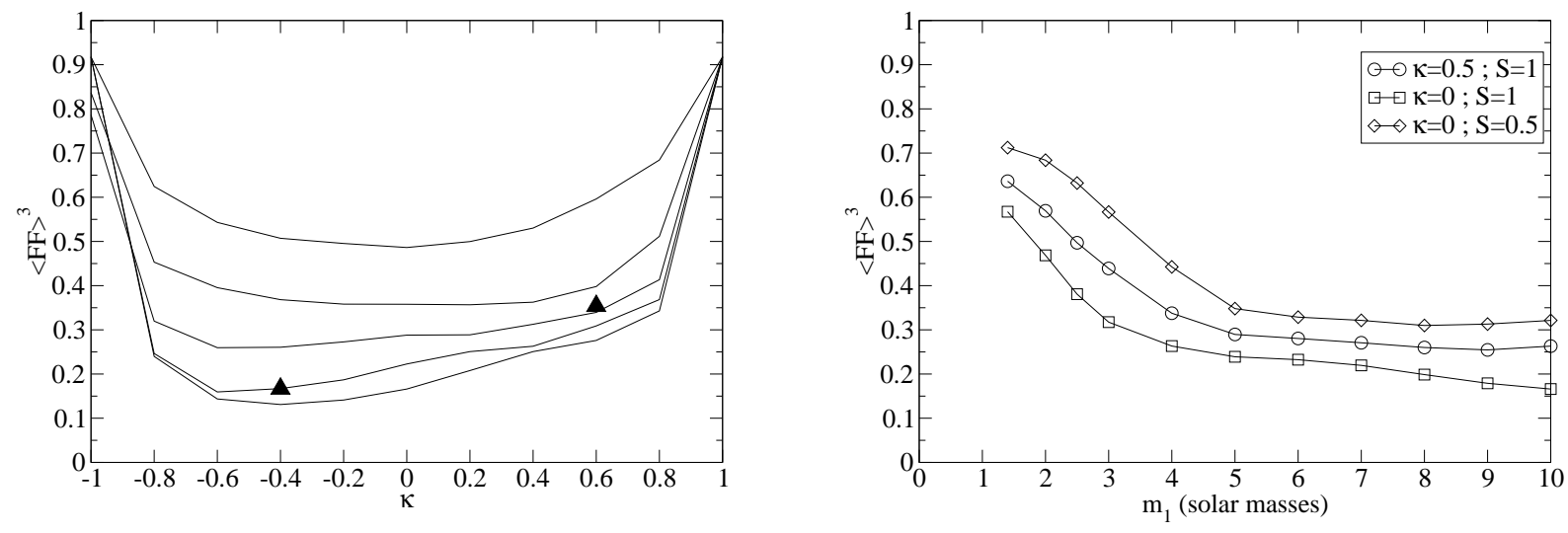

FIG. 1: The left panel shows the value of $\left\langle\mathrm{FF}>^{3}\right.$ as a function of $\kappa$, the cosine of the spin tilt angle. The masses are $m_{1}=10 M_{\odot}$ and $m_{2}=1.4 M_{\odot} . S$ varies from 1 (lower curve) to 0.2 (higher curve), with a step of 0.2 . The solid triangles denote the results including $2 \mathrm{PN}$ corrections to the non-precessing parts, for $S=1$. The right panel shows the dependence of the reduced detection rate on the mass of the spinning object $m_{1} . m_{2}$ is fixed to $1.4 \mathrm{M}_{\odot}$. Results are shown for 3 different sets of spin properties.
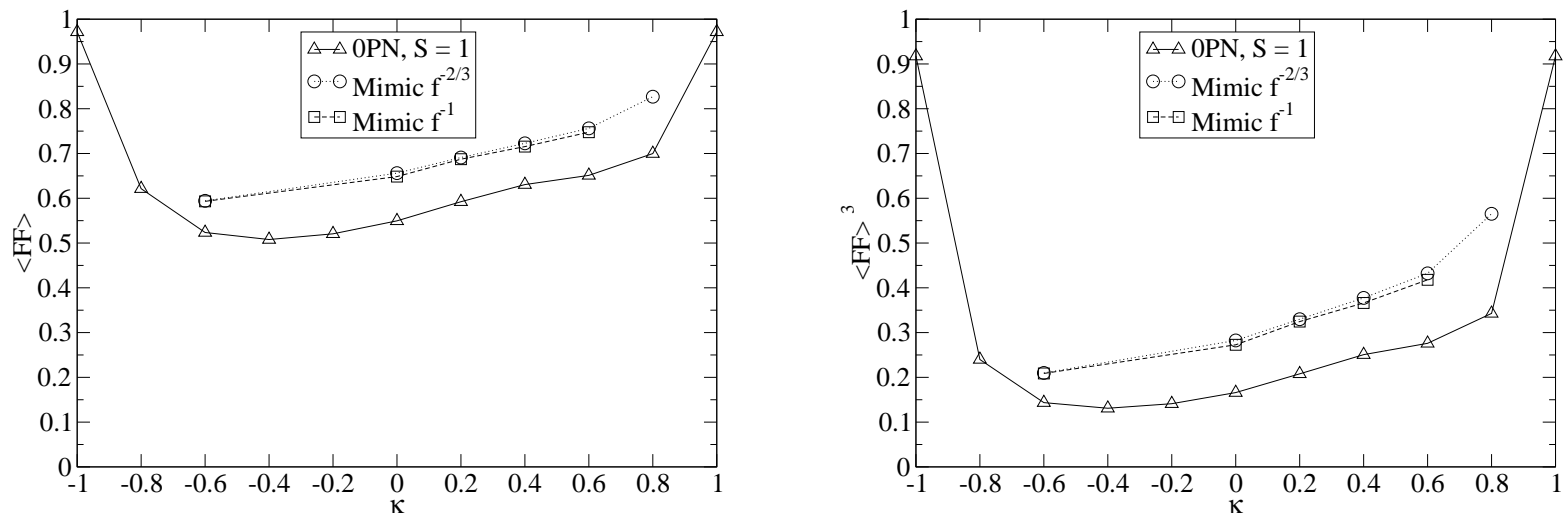

FIG. 2: Efficiency of the "mimic" templates in recovering the signal-to-noise ratio and increasing the inspiral detection rate. The triangles and solid lines correspond the results obtained using the chirp templates without any precession modification (2). The circles and dotted line denote results when the phase correction is included with the $-2 / 3$ exponent, and the squares and dashed line with the -1 exponent. The left panel shows the average $\langle\mathrm{FF}\rangle$ whereas the right panel shows the reduction factors of the detection rate, as a function of the cosine of the spin-misalignment angle. The masses are $m_{1}=10 M_{\odot}$ and $m_{2}=1.4 M_{\odot}$ and the spin magnitude is maximum $(S=1)$.

in Sec. II C, and in comparison to results with templates that do not include any precession-like modification. As mentioned above, the results do not vary significantly with the frequency exponent : results obtained using the $-2 / 3$ and -1 are almost identical. As expected there is an improvement of the fitting factor, which is in the range 15-30\%. Nevertheless, we are far from recovering a high SNR. The detection rate can still reduced by up $80 \%$. For the other pairs of masses we have examined, fitting factors increase by about 10\%-20\% for the full range of spin properties. Based on these results we conclude that the "mimic" phase correction proposed by [1] alone is not enough to raise the detection efficiency to desirable levels. 


\section{CONCLUSION}

In this paper we present a systematic and quantitative study of the effect of precession on the detection of binary inspiral signals. Unlike earlier work [1], we focus on (i) implications for the initial LIGO observations, (ii) the signalto-noise ratio reduction due to the effects of precession alone - by analyzing signals and templates computed at the same PN order for the non-precessing portion of the phase - and (iii) the quantitative study of the fitting factor of a family of mimic templates that have been suggested for recovering high SNR. We have examined results for 4 pairs of masses for the full range of spin properties and directions, and discussed in more detail the results of one of these pairs $((10 ; 1.4)$ solar masses $)$ that exhibits the strongest precession effects and is appropriate for binaries with a black hole and a neutron star.

We first addressed the question of how important is precession and for what binary properties. We found that it can seriously affect detection, if mass ratios are in excess of about 2 , spin magnitudes in excess of about $30 \%$ of maximum, and spin tilts in excess of about 35 degrees. These results help in the identification of the parameter space where ways of improving the detection efficiency must be found. We found that the detection rate can decrease by almost an order of magnitude if searches are performed with templates that do not include precession effects. Such a loss of events can be very concerning, given the current low-estimates for the expected detection rates [15].

Precession waveforms depend on a large number of parameters and their use as a template family is not feasible. Therefore, it is important to introduce a family of templates that can "mimic" precession effects well enough, but they depend on a small number of parameters. Here we tested one such family suggested in [1], and found that, although they do increase the detection rate, this increase is not significant enough to raise the detection rate to desirable levels (leading to improvement of more than a factor of a few for the case of strong precession). We conclude that other forms of "mimic" templates must be explored in the near future. Such an exploration is beyond the scope of the present paper, but we are undertaking it as the next step in this project.

One can further restrict the physical parameter space over which precession is crucial for detection, by convolving our results from the first part of the paper with astrophysically relevant distributions of binary and spin parameters of double compact objects, derived based on our current understanding of the formation of compact binaries with black holes. We are currently working on such a convolution with detailed population calculations for many formation models [15] of BH binaries, with the goal of obtaining an astrophysically-motivated picture of how important it will be to include precession in the template families for the search of GW inspiral signals in the next few years [28].

\section{Acknowledgments}

We would like to thank B. Allen, P. Brady, A. Buonanno, and B. Owen for useful discussions. This work is supported by NSF Grant PHY-0121420. We are also grateful to the High Energy Physics group at Northwestern University for allowing us to access their computer cluster THEMIS.

[1] T.A. Apostolatos, Phys. Rev. D 54, 2421 (1996).

[2] A. Abramovici et al., Science 256, 325 (1992).

[3] B. Caron et al., Nucl. Phys. B54, 167 (1997).

[4] K. Danzmann, in Gravitational Waves Experiments, eds. E. Coccia, G. Pizzella and F. Ronga, World Scientific, Singapore (1995).

[5] H. Tagoshi et al., Phys. Rev. D. 63, 062001 (2001).

[6] C.W. Helstrom, Statistical Theory of Signal Detection, 2nd edition, Pergamon Press, London (1968).

[7] B.J. Owen and B.S. Sathyaprakash, Phys. Rev. D 60, 022002 (1999).

[8] C. Cutler and K. S. Thorne, An Overview of Gravitational-Wave Sources, to appear in Proceedings of GR16 (Durban, South Africa, 2001), gr-qc/0204090.

[9] L. Blanchet, Living Review in Relativity 2002-3 http://www.livingreviews.org/Articles/Volume5/20023blanchet/index.html (2002)

[10] T. Damour, B. R. Iyer and B. S. Sathyaprakash, Phys. Rev. D 57, 885 (1998).

[11] T. Damour, B. R. Iyer and B. S. Sathyaprakash, Phys. Rev. D 62, 084036 (2000).

[12] T.A. Apostolatos, C. Cutler, G.J. Sussman and K.S. Thorne, Phys. Rev. D 49, 6274 (1994).

[13] T.A. Apostolatos, Phys. Rev. D 52, 605 (1995).

[14] V. Kalogera and K. Belczynski, to appear in AIP proceedings Astrophysical Sources of Gravitational Radiation for GroundBased Detectors., astro-ph/0101047 (2001).

[15] K. Belczynski, V. Kalogera and T. Bulik, ApJ 572, in press (2002).

[16] J.H. Taylor and J.M. Weisberg, APJ 253, 908 (1982). 
[17] A. Wolszczan, Nature 350, 688 (1991).

[18] L. Bildsten and C. Cutler, ApJ 400, 175 (1992).

[19] C.S. Kochanek, ApJ 398, 234 (1992).

[20] L.E. Kidder, Phys. Rev. D 52, 821 (1995).

[21] P.R. Brady, J.D.E. Creighton and K.S. Thorne, Phys. Rev. D 58, 061501 (1998).

[22] A. Buonanno, Y. Chen and M. Vallisneri, Phys. Rev. D submitted, gr-qc/0205122 (2002).

[23] B.S. Sathyaprakash and S.V. Dhurandhar, Phys. Rev. D 44, 3819 (1991).

[24] S.V. Dhurandhar and B.S. Sathyaprakash, Phys. Rev. D 49, 1707 (1994).

[25] R. Balasubramanian and S.V. Dhurandhar, Phys. Rev. D 50, 6080 (1994).

[26] C. Cutler and E.E. Flanagan, Phys. Rev. D 49, 2658 (1994).

[27] L.S. Finn, Written version of lectures given at XXVI SLAC Summer Institute on Particle Physics Gravity: From the Hubble Length to the Planck Length, gr-qc/9903107, (1998).

[28] C.M. Ihm, V. Kalogera, P. Grandclement and C. Belczynski, in prep. 